\title{
QUALIDADE FÍSICA DE UM LATOSSOLO VERMELHO SUBMETIDO A SISTEMAS DE MANEJO AVALIADO PELO ÍNDICE $\mathrm{S}^{(1)}$
}

\author{
Fabiana de Souza Pereira ${ }^{(2)}$, Itamar Andrioli ${ }^{(3)}$, Faber de Souza \\ Pereira $^{(4)}$, Paula Regina de Oliveira ${ }^{(5)}$, José Frederico Centurion ${ }^{(3)}$, \\ Ricardo Jorge Falqueto ${ }^{(6)} \&$ Alba Leonor da Silva Martins ${ }^{(2)}$
}

\begin{abstract}
RESUMO
O monitoramento da qualidade do solo pelos atributos físicos é importante para a avaliação e manutenção da sustentabilidade dos sistemas agrícolas. Um atributo indicador da qualidade do solo deve ser sensível às variações do manejo ao qual está sendo submetido. Este estudo teve como objetivo avaliar o parâmetro $\mathrm{S}$ como indicador da qualidade física do solo e a agregação de um Latossolo Vermelho distrófico, submetido a sistemas de manejo sem ou com a inclusão de plantas de cobertura em pré-safra durante 11 anos. Os tratamentos foram: sistema convencional (SC) e sistemas conservacionistas compostos por plantas de cobertura, crotalária (SDC), milheto (SDM) e lablabe (SDL). O delineamento experimental foi o de blocos casualizados, com quatro tratamentos e seis repetições; as camadas constituíram-se nas parcelas subdivididas. Os sistemas conservacionistas SDC, SDM e SDL apresentaram maior teor de matéria orgânica em relação ao $\mathrm{SC}$ nas camadas de 0 a $0,05 \mathrm{~m}$. Maiores valores de diâmetro médio
\end{abstract}

\footnotetext{
(1) Recebido para publicação em março de 2010 e aprovado em novembro de 2010.

(2) Doutoranda do Departamento de Solos e Adubos da Faculdade de Ciências Agrárias e Veterinárias, Universidade Estadual Paulista - FCAV/UNESP. Via de Acesso Professor Paulo Donato Castellane, s/n, CEP 14884-900 Jaboticabal (SP). E-mail: fabianadsp@hotmail.com; albaleonor@ibest.com.br

(3) Professor adjunto do Departamento de Solos e Adubos da Faculdade de Ciências Agrárias e Veterinárias, Universidade Estadual Paulista - FCAV/UNESP. E-mail: Itamar@fcav.unesp.br; jfcentur@fcav.unesp.br

(4) Engenheiro-Agrônomo formado pela Universidade Estadual de Goiás - UEG. Rod. GO 330, km 241 - Anel Viário, CEP 75780 000 Ipameri (GO). E-mail: faberfla@hotmail.com

(5) Mestranda do Departamento de Solos e Adubos da Faculdade de Ciências Agrárias e Veterinárias, Universidade Estadual Paulista - FCAV/UNESP. E-mail: paularoliver@hotmail.com

(6) Doutor do Departamento de Solos e Adubos da Faculdade de Ciências Agrárias e Veterinárias, Universidade Estadual Paulista - FCAV/UNESP. E-mail: jrfalqueto@yahoo.com.br
} 


\begin{abstract}
ponderado e geométrico dos agregados foram observados nos sistemas conservacionistas. $\mathrm{O}$ solo apresentou boa qualidade física determinada pelo índice $\mathrm{S}$, com valores todos superiores a 0,035 .
\end{abstract}

Termos de indexação: atributos físicos, agregados, plantas de cobertura.

\title{
SUMMARY: PHYSICAL QUALITY OF AN OXISOL UNDER MANAGEMENT SYSTEMS EVALUATED BY THE INDEX "S"
}

\begin{abstract}
The monitoring of soil quality based on physical properties is important for the evaluation and maintenance of the sustainability of agricultural systems. An indicator property of soil quality should be sensitive to management changes. This study aimed to evaluate the $S$ parameter as an indicator of soil physical quality and aggregation of an Oxisol, managed for 11 years in systems with and without the inclusion of off-season cover crops. The treatments consisted of: conventional system (SC) and conservation systems with cover crops (sunn hemp - SDC, millet-SDM, and lab-lab-SDL). The experimental design was a randomized block design with four treatments and six replications, the split plots consisted of soil layers. The organic matter content in the conservation systems SDC, SDM and SDL was higher than in the conventional system in the 0-0.05m layer. Higher mean values of weighted and geometric diameter of the aggregates were observed in the conservation tillage systems. The $S$ index indicated good physical quality (all values $>0.035$ ).
\end{abstract}

Index terms: physical properties, aggregates, cover crops.

\section{INTRODUÇÃO}

O preparo do solo tem como finalidade proporcionar condições favoráveis ao adequado crescimento e desenvolvimento das culturas (Furlani et al., 2005). O cultivo intensivo do solo e seu preparo em condições inadequadas alteram os fatores de crescimento das culturas. Essas alterações são mais pronunciadas nos sistemas de preparo onde há o revolvimento do solo do que nos conservacionistas; elas manifestam-se nas modificações que ocorrem na estrutura do solo, que são evidenciadas por alterações nos valores de densidade do solo, resistência mecânica à penetração, porosidade total, porosidade de aeração, armazenagem e disponibilidade de água às plantas (Klein, 1998).

No âmbito agropecuário, o sistema plantio direto tem sido uma das melhores alternativas para a manutenção da sustentabilidade dos recursos naturais na utilização dos solos (Oliveira et al., 2002). Por apresentar benefício ambiental amplo, é possível que esse sistema seja a contribuição mais importante que a agricultura está oferecendo em termos de preservação ambiental (Wietholter et al., 1998).

Estudos demonstram os efeitos benéficos das plantas de cobertura nas propriedades do solo e no rendimento das culturas, decorrentes principalmente da produção de fitomassa, do acúmulo e da posterior liberação de nutrientes, pela decomposição da palhada (Giacomini, 2003; Espíndola et al., 2006; Boer et al., 2007).
Em relação às propriedades físicas do solo, as plantas de cobertura e o seu tempo de utilização apresentam melhoria da estrutura do solo (Wendling et al., 2005), a qual, segundo Letey (1985), exerce grande influência na movimentação e infiltração de água, transferência de calor e aeração. Por sua vez, a melhor qualidade estrutural do solo coincide com maior agregação.

A qualidade física do solo é fundamental para a sustentabilidade dos sistemas agropecuários e deve ser avaliada por meio de atributos que descrevem o seu comportamento.

Dexter (2004) sugeriu a utilização do índice S, que é definido como a inclinação da curva de retenção de água no ponto de inflexão. Valores de $\mathrm{S}$ maiores do que 0,035 foram estabelecidos como favoráveis ao crescimento das raízes, e abaixo desse valor, como restritivos. Ou seja, valores $>0,035$ indicam adequada distribuição de tamanho de poros e qualidade física do solo, independentemente do tipo deste, em clima temperado. Dessa forma, fatores que alteram a distribuição do diâmetro dos poros, como o uso e manejo do solo, podem ser avaliados e comparados diretamente por esse índice.

De acordo com Tormena et al. (2008), o índice S diferenciou os sistemas de preparo e posições de amostragem, sendo $\mathrm{S}_{\mathrm{PD}}<\mathrm{S}_{\mathrm{PC}}$ (índice $\mathrm{S}$ em plantio direto e índice $\mathrm{S}$ em plantio convencional, respectivamente), independentemente das posições de amostragem linha e entrelinha. Na posição linha 
verificaram-se maiores valores de $\mathrm{S}$ tanto em $\mathrm{PD}$ como em PC. O valor de $\mathrm{S}$ diminuiu com o aumento da densidade do solo, indicando redução de sua qualidade física.

Silva et al. (2008) mostraram que a área de mata nativa apresentou melhor qualidade física do solo determinada pelo índice $\mathrm{S}$, em relação às áreas de plantio direto.

Marchão et al. (2007) verificaram que o índice S variou em função do sistema de integração lavourapecuária e da profundidade de amostragem, demonstrando ser um índice sensível às alterações na estrutura do solo. Os resultados confirmam a hipótese estabelecida e sugerem que mais estudos devem ser conduzidos para quantificar o índice $\mathrm{S}$ em diferentes solos e sistemas de manejo sob condições tropicais.

Este estudo teve como objetivo avaliar o índice $\mathrm{S}$ como indicador da qualidade física do solo e a agregação de um Latossolo Vermelho distrófico, submetido ao sistema de preparo convencional do solo e ao sistema de plantio direto com inclusão de plantas de cobertura em pré-safra durante 11 anos.

\section{MATERIAL E MÉTODOS}

O estudo foi realizado em Jaboticabal, SP ( $21^{\circ} 15^{\prime} \mathrm{S}$ e $48^{\circ} 18^{\prime} \mathrm{W} ; 595 \mathrm{~m}$ de altitude), em uma área com 11 anos de adoção de sistemas de manejo do solo. O clima é do tipo Cwa, segundo a classificação de Köppen, com temperaturas moderadas (média de $22,4^{\circ} \mathrm{C}$ ), verão quente e chuvoso e precipitação pluvial média anual de $1.285 \mathrm{~mm}$.

A área do experimento é representada por um Latossolo Vermelho distrófico textura média típico (LVd) (Andrioli \& Centurion, 1999), cuja composição granulométrica foi determinada na camada de 0 a 0,20 $\mathrm{m}$ em amostras deformadas por meio da dispersão com $\mathrm{NaOH}\left(0,1 \mathrm{~mol} \mathrm{~L}^{-1}\right)$ e agitação lenta durante $16 \mathrm{~h}$, sendo o conteúdo de argila obtido pelo método da pipeta (Gee \& Bauder, 1986). A granulometria do LVd apresentou $310 \mathrm{~g} \mathrm{~kg}^{-1}$ de argila, $48 \mathrm{~g} \mathrm{~kg}^{-1}$ de silte e $642 \mathrm{~g} \mathrm{~kg}^{-1}$ de areia.

O delineamento experimental foi o de blocos casualizados, com parcelas de 4,5 m de largura e $20 \mathrm{~m}$ de comprimento, com quatro tratamentos e seis repetições com parcelas subdivididas, sendo estas formadas pelas camadas analisadas.

A área experimental foi cultivada desde 1995 com plantas de cobertura em pré-safra (setembro a dezembro), nos sistemas de semeadura direta e convencional. As culturas anuais foram o milho, durante os anos de 1995 a 1997, a soja, entre 1998 e 2000, e o milho, de 2001 a 2007. Desde o ano de 2001 a área vem sendo cultivada anualmente com milho (Zea mays), sob o sistema de semeadura direta com as seguintes plantas de cobertura: crotalária (Crotalaria juncea) (SDC), milheto (Pennisetum americanum) (SDM) e lablabe (Dolechus lablab) (SDL) e sistema convencional (gradagem aradora e duas niveladoras) (SC).

A semeadura das plantas de cobertura foi realizada até na segunda quinzena de setembro, após as primeiras chuvas. Utilizou-se de uma semeadora de plantio direto provida de sulcador, no espaçamento de 0,45 m entre linhas, e 20, 30 e $50 \mathrm{~kg} \mathrm{ha}^{-1}$ de sementes de milheto, lablabe e crotalária, respectivamente. No início de dezembro foram realizadas as dessecações, mediante a aplicação de herbicida glifosate $\left(2,4 \mathrm{~kg} \mathrm{ha}^{-1}\right.$ de i.a.); em seguida, foram realizadas as semeaduras de milho tanto no sistema convencional quanto no direto.

Foram coletadas, nas camadas de 0 a 0,05, 0,05 a 0,10, 0,10 a 0,15 e 0,15 a 0,20 m, amostras deformadas para determinação do conteúdo da matéria orgânica, conforme método descrito por Raij et al. (1987).

A avaliação da estabilidade de agregados foi feita segundo Kemper \& Rosenau (1986), nas profundidades de 0 a 0,05 e 0,05 a $0,10 \mathrm{~m}$. O diâmetro médio geométrico (DMG) e o diâmetro médio ponderado (DMP) foram calculados conforme Kemper \& Chepil (1965).

Para determinação das propriedades físicas do solo, foram coletadas amostras indeformadas do solo nas entrelinhas da cultura, nos sistemas de manejo estudados nas camadas de 0 a 0,05, 0,05 a 0,10, 0,10 a 0,15 e 0,15 a 0,20 m. Após devidamente preparadas, as amostras foram saturadas por meio de elevação gradual de uma lâmina de água em uma bandeja e, em seguida, submetidas às tensões de 0,001, 0,006, 0,01, 0,033, 0,06 e 0,3 MPa, em câmaras de Richards (Klute, 1986). Ao atingirem o equilíbrio, foram pesadas e, na sequência, secas em estufas a $105^{\circ} \mathrm{C}$ durante $24 \mathrm{~h}$, para determinação do conteúdo de água em cada tensão (Gardner, 1986) e da densidade do solo (Blake \& Hartge, 1986). A microporosidade foi determinada por secagem na tensão de 0,006 MPa; a porosidade total, segundo Danielson \& Sutherland (1986); e a macroporosidade, obtida por diferença entre a porosidade total e a microporosidade.

As curvas de retenção de água foram ajustadas pelo modelo proposto por van Genuchten (1980), a partir do qual foi obtida a inclinação e, consequentemente, o índice $\mathrm{S}$, como se segue:

$$
\mathrm{S}=-\mathrm{n}\left(\theta_{\text {sat }}-\theta_{\text {res }}\right)[1+1 / \mathrm{m}]^{-(1+\mathrm{m})}
$$

em que: $n$ e m são parâmetros que governam o formato da curva; e $\theta_{\text {sat }}$ e $\theta_{\text {res, }}$ a umidade na saturação e residual, respectivamente.

A adubação do milho foi feita com $350 \mathrm{~kg}$ da fórmula 4-20-20 (NPK), e a produtividade de grãos de milho foi determinada na safra 2007/2008, em uma área de $22,5 \mathrm{~m}^{2}$. A umidade dos grãos foi corrigida para $0,13 \mathrm{~kg} \mathrm{~kg}^{-1}$. 
Os resultados foram submetidos à análise de variância e, quando esta indicou diferença entre médias, foi utilizado o teste de Tukey a $5 \%$.

\section{RESULTADOS E DISCUSSÃO}

Os sistemas de semeadura direta, com cultivo de crotalária (SDC), milheto (SDM) e lablabe (SDL), em pré-safra, apresentaram maior valor de matéria orgânica na camada de 0 a 0,05 m e não diferiram entre si (Quadro 1). Segundo Souza Neto et al. (2008), o menor revolvimento do solo no sistema semeadura direta reduz o contato do solo com os materiais vegetais, diminuindo assim a velocidade de decomposição, quando comparado ao SC, no qual ocorre revolvimento do solo, fragmentação, incorporação e exposição dos restos vegetais à ação mais intensa dos microrganismos que atuam na sua decomposição.

Dessa forma, a substituição do preparo convencional pelo plantio direto durante 11 anos resultou numa recuperação dos teores de matéria orgânica.

Albuquerque et al. (2005) verificaram, em um Latossolo Vermelho distroférrico muito argiloso, que as plantas de cobertura aumentaram o teor de matéria orgânica. Segundo esses pesquisadores, em períodos mais longos, as plantas de cobertura, associadas aos preparos conservacionistas, pelo aumento da matéria orgânica, podem melhorar as características físicas do solo.

Araújo et al. (2004) observaram que as modificações nos teores de matéria orgânica do solo estão associadas a diferentes fatores, em especial à intensidade de revolvimento do solo, podendo ter reflexos na forma e estabilidade da estrutura, com consequência no comportamento físico do solo.

Os tratamentos diferiram quanto à macroporosidade somente na camada de 0 a $0,05 \mathrm{~m}$, em que os maiores valores foram verificados no sistema de semeadura direta lablabe (SDL) e no sistema convencional (SC); este não diferiu do sistema direto crotalária (SDC) (Quadro 2). Os valores altos de macroporosidade no SC devem-se à maior mobilização nas camadas superficiais; nas demais camadas, os tratamentos não diferiram.

O SDL teve menor macroporosidade nas camadas inferiores em relação à de 0 a $0,05 \mathrm{~m}$. Logo, o SDM não diferiu entre as camadas. Apesar do maior conteúdo de matéria orgânica nos sistemas de semeadura direta, na camada de 0-0,05 m ele não resultou na melhoria da macroporosidade, exceto para o SDL.

O SC apresentou maior macroporosidade nas duas primeiras camadas $(0$ a 0,05 e 0,05 a $0,10 \mathrm{~m}$ ). Segundo Cruz et al. (2003), esse fato deve-se certamente às operações de preparo do solo. Schafer et al. (2001) constataram que o uso da grade pesada resultou em diminuição aparente da macroporosidade e em aumento do adensamento na superfície do solo, evidenciando a presença de "pé de grade".

A microporosidade foi maior no SDM em relação aos outros tratamentos na camada de 0 a $0,05 \mathrm{~m}$; esse sistema foi o que teve menor valor de macroporosidade nessa camada. O SC teve aumento considerável da microporosidade na camada de 0 a $0,05 \mathrm{~m}$ em relação à de 0,15 a $0,20 \mathrm{~m}$. O SDC e o SDM apresentaram diminuição da microporosidade de 10 e $24 \%$, respectivamente, entre essas camadas.

A porosidade total foi maior nos sistemas de semeadura direta; com o aumento da profundidade, os seus valores diminuíram em todos os tratamentos.

Os sistemas conservacionistas tiveram maiores valores de diâmetro médio ponderado (DMG) em relação ao sistema convencional. Beutler et al. (2001), em Latossolo Vermelho distrófico textura argilosa, observaram aumento do DMG em sistemas conservacionistas. Resultados semelhantes foram encontrados por Costa et al. (2003) em Latossolo Bruno em Guarapuava (PR), onde observaram aumento do

Quadro 1. Teores de matéria orgânica em diferentes tratamentos e camadas de um Latossolo Vermelho distrófico

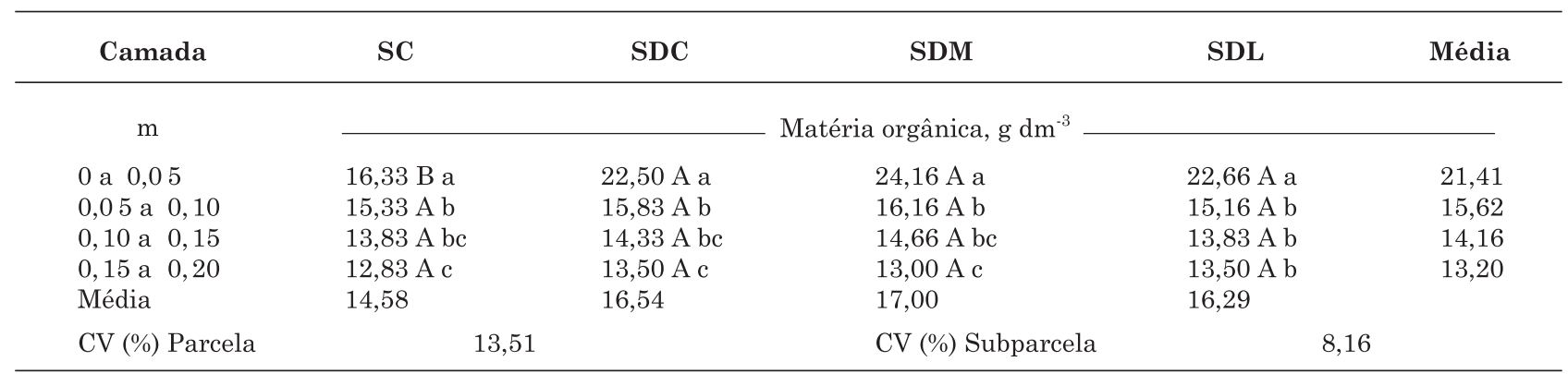

SC: semeadura convencional; SDC: semeadura direta crotalária; SDM: semeadura direta milheto; SDL: semeadura direta lablabe. Médias seguidas da mesma letra não diferem estatisticamente pelo teste de Tukey a 5\%. Letras maiúsculas comparam as médias na linha, e minúsculas, na coluna. 
Quadro 2. Valores de porosidade total, macro e microporosidade do solo nos sistemas de manejo para a camada de 0 a $0,20 \mathrm{~m}$

\begin{tabular}{|c|c|c|c|c|c|}
\hline Camada & SC & SDC & SDM & SDL & Média \\
\hline \multicolumn{6}{|c|}{ Macroporosidade $\left(\mathrm{m}^{3} \mathrm{~m}^{-3}\right)$} \\
\hline 0,0 a 0,05 & $0,15 \mathrm{AB}$ a & $0,12 \mathrm{BC} \mathrm{ab}$ & $0,10 \mathrm{C} \mathrm{a}$ & $0,18 \mathrm{~A} \mathrm{a}$ & 0,14 \\
\hline 0,05 a 0,10 & $0,14 \mathrm{~A} \mathrm{a}$ & $0,15 \mathrm{~A} \mathrm{a}$ & $0,14 \mathrm{~A} \mathrm{a}$ & $0,12 \mathrm{~A} \mathrm{~b}$ & 0,14 \\
\hline 0,10 a 0,15 & $0,09 \mathrm{~A} \mathrm{~b}$ & $0,11 \mathrm{~A} \mathrm{~b}$ & $0,10 \mathrm{~A} \mathrm{a}$ & $0,08 \mathrm{~A} \mathrm{~b}$ & 0,09 \\
\hline 0,15 a 0,20 & $0,09 \mathrm{~A} \mathrm{~b}$ & $0,13 \mathrm{~A} \mathrm{ab}$ & $0,12 \mathrm{~A} \mathrm{a}$ & $0,11 \mathrm{~A} \mathrm{~b}$ & 0,11 \\
\hline Média & 0,12 & 0,13 & 0,11 & 0,12 & \\
\hline CV (\%) Parcela & & 15,88 & $\mathrm{CV}(\%) \mathrm{S}$ & & 22,3 \\
\hline \multicolumn{6}{|c|}{ Microporosidade $\left(\mathrm{m}^{3} \mathrm{~m}^{-3}\right)$} \\
\hline 0,0 a 0,05 & $0,25 \mathrm{C} \mathrm{b}$ & $0,30 \mathrm{~B} \mathrm{a}$ & $0,33 \mathrm{~A} \mathrm{a}$ & $0,27 \mathrm{C} \mathrm{b}$ & 0,29 \\
\hline 0,05 a 0,10 & $0,25 \mathrm{~A} \mathrm{~b}$ & $0,26 \mathrm{~A} \mathrm{~b}$ & $0,25 \mathrm{Ac}$ & $0,26 \mathrm{~A} \mathrm{~b}$ & 0,25 \\
\hline 0,10 a 0,15 & $0,30 \mathrm{~A} \mathrm{a}$ & $0,30 \mathrm{~A} \mathrm{a}$ & $0,30 \mathrm{~A} \mathrm{~b}$ & $0,29 \mathrm{~A} \mathrm{a}$ & 0,30 \\
\hline 0,15 a 0,20 & $0,28 \mathrm{~A} \mathrm{a}$ & $0,27 \mathrm{AB} \mathrm{b}$ & $0,25 \mathrm{~B} \mathrm{c}$ & $0,27 \mathrm{AB} \mathrm{b}$ & 0,27 \\
\hline Média & 0,27 & 0,28 & 0,28 & 0,27 & \\
\hline CV (\%) Parcela & & 5,07 & $\mathrm{CV}(\%) \mathrm{S}$ & & 5,42 \\
\hline \multicolumn{6}{|c|}{ Porosidade total $\left(\mathrm{m}^{3} \mathrm{~m}^{-3}\right)$} \\
\hline 0,0 a 0,05 & $0,37 \mathrm{~B} \mathrm{~b}$ & $0,43 \mathrm{~A} \mathrm{a}$ & $0,44 \mathrm{~A} \mathrm{a}$ & $0,45 \mathrm{~A} \mathrm{a}$ & 0,42 \\
\hline 0,05 a 0,10 & $0,41 \mathrm{~A} \mathrm{a}$ & $0,40 \mathrm{AB} a b$ & $0,40 \mathrm{AB}$ & $0,37 \mathrm{~B} \mathrm{~b}$ & 0,39 \\
\hline 0,10 a 0,15 & $0,39 \mathrm{~A} \mathrm{ab}$ & $0,38 \mathrm{~A} \mathrm{~b}$ & $0,39 \mathrm{~A} \mathrm{~b}$ & $0,38 \mathrm{~A} \mathrm{~b}$ & 0,38 \\
\hline 0,15 a 0,20 & $0,37 \mathrm{~A} \mathrm{~b}$ & $0,40 \mathrm{~A} \mathrm{ab}$ & $0,38 \mathrm{~A} \mathrm{~b}$ & $0,37 \mathrm{~A} \mathrm{~b}$ & 0,38 \\
\hline Média & 0,38 & 0,40 & 0,40 & 0,39 & \\
\hline CV (\%) Parcela & & 3,81 & $\mathrm{CV}(\%) \mathrm{S}$ & & 5,96 \\
\hline \multicolumn{6}{|c|}{ Diâmetro Médio Geométrico dos Agregados (DMG) (mm) } \\
\hline 0,0 a 0,05 & $2,73 \mathrm{~B}$ a & $4,84 \mathrm{~A} \mathrm{a}$ & $5,31 \mathrm{~A} \mathrm{a}$ & $5,51 \mathrm{~A} \mathrm{a}$ & 4,60 \\
\hline 0,05 a 0,10 & $2,53 \mathrm{~B} \mathrm{a}$ & $3,37 \mathrm{~A} \mathrm{a}$ & $4,05 \mathrm{~A} \mathrm{a}$ & $4,92 \mathrm{~A} \mathrm{a}$ & 3,97 \\
\hline Média & 2,63 & 4,60 & 4,68 & 5,21 & \\
\hline CV (\%) Parcela & & 16,01 & $\mathrm{CV}(\%) \mathrm{Su}$ & & 15,40 \\
\hline \multicolumn{6}{|c|}{ Diâmetro Médio Ponderado dos Agregados (DMP) (mm) } \\
\hline 0,0 a 0,05 & $2,65 \mathrm{~B} \mathrm{a}$ & $5,41 \mathrm{~A} \mathrm{a}$ & $5,66 \mathrm{~A} \mathrm{a}$ & $5,34 \mathrm{~A} \mathrm{a}$ & 4,76 \\
\hline 0,05 a 0,10 & $2,52 \mathrm{~B} \mathrm{a}$ & $4,74 \mathrm{~A} \mathrm{a}$ & $4,92 \mathrm{~A} \mathrm{a}$ & $5,16 \mathrm{Aa}$ & 4,33 \\
\hline Média & 2,58 & 5,07 & 5,29 & 5,25 & \\
\hline CV (\%) Parcela & & 9,94 & $\mathrm{CV}(\%) \mathrm{S}$ & & 9,16 \\
\hline
\end{tabular}

Médias seguidas da mesma letra não diferem estatisticamente pelo teste de Tukey a $5 \%$. Letras maiúsculas comparam as médias na linha, e minúsculas, na coluna. SC: semeadura convencional; SDC: semeadura direta crotalária; SDM: semeadura direta milheto; SDL: semeadura direta lablabe.

DMG na camada de 0 a $0,05 \mathrm{~m}$ - de $1,6 \mathrm{~mm}$ em $\mathrm{SC}$ para 3,7 mm nos sistemas conservacionistas - após 21 anos de instalação do experimento.

Verifica-se, que o DMP foi maior nas áreas de sistemas conservacionistas em relação às áreas sob sistema convencional (Quadro 2), demonstrando que 11 anos após a implantação do sistema foram suficientes para demonstrar seu aumento. Resultados semelhantes foram obtidos por Silva et al. (2008) após seis anos da implantação do sistema plantio direto.

Não houve interação entre os sistemas de manejo e as camadas analisadas em relação à densidade do solo (Quadro 3). O SC teve menor valor de densidade do solo em relação aos sistemas de semeadura direta, não diferindo do SDC. Os menores valores da densidade do solo podem ser atribuídos ao intenso revolvimento do solo e à incorporação de resíduos culturais. Centurion \& Demattê (1985) também observaram maior valor de densidade do solo no sistema de semeadura direta, comparado com outros sistemas de preparo. Entre os sistemas de manejo estudados, a camada de 0 a 0,05 $\mathrm{m}$ foi a que teve a menor densidade do solo; a camada de 0,05 a 0,10 m não diferiu da camada de 0,15 a $0,20 \mathrm{~m}$.

Na figura 1 são apresentadas as curvas de retenção de água no Latossolo Vermelho distrófico ( $\mathrm{LVd}$ ) nos sistemas de manejo e nas diferentes camadas, ajustadas segundo o modelo matemático proposto por van Genuchten (1980).

$\mathrm{Na}$ tensão de $0,01 \mathrm{MPa}$, correspondente à capacidade de campo, todas as camadas e sistemas de manejo tiveram retenção de água semelhante, exceto o SDM, que seguiu o volume de microporos apresentado, ou seja, esse tratamento teve a maior retenção de água nessa tensão devido à maior microporosidade observada na camada de 0 a $0,05 \mathrm{~m}$, 
Quadro 3. Densidade do solo nos sistemas de manejo para a camada de 0 a $0,20 \mathrm{~m}$

\begin{tabular}{lc}
\hline Sistemas (S) & Densidade do solo $\left(\mathbf{m g ~ m}^{-3}\right)$ \\
\hline SC & $1,26 \mathrm{~b}$ \\
SDC & $1,28 \mathrm{ab}$ \\
SDM & $1,32 \mathrm{a}$ \\
SDL & $1,31 \mathrm{a}$ \\
Teste F & $5,84^{* *}$ \\
DMS (5\%) & 0,045 \\
Camadas (C) & \\
0 a 0,05 & $1,15 \mathrm{c}$ \\
0,05 a 0,10 & $1,33 \mathrm{ab}$ \\
0,10 a 0,15 & $1,32 \mathrm{~b}$ \\
0,15 a 0,20 & $1,38 \mathrm{a}$ \\
Teste F & $40,06^{* *}$ \\
DMS (5\%) & 0,060 \\
Interação S x C & $0,82^{\text {ns }}$ \\
\hline
\end{tabular}

Médias seguidas da mesma letra não diferem estatisticamente pelo teste de Tukey a $5 \%$. SC: semeadura convencional; SDC: semeadura direta crotalária; SDM: semeadura direta milheto; SDL: semeadura direta lablabe.

concordando com Araújo et al. (2004), os quais também constataram que o aumento da água retida deveu-se à alteração na distribuição do tamanho de poros, com o aumento de poros de menor diâmetro.

O SDC apresentou maior retenção de água na camada de 0,10 a $0,15 \mathrm{~m}$, devido provavelmente a maior densidade do solo, concordando com Araújo et al. (2004), que também constataram que o aumento da densidade do solo resultou em aumento da água retida.

As variações observadas entre as curvas de retenção de água apresentadas nos sistemas conservacionistas podem ser relacionadas à influência da composição dos materiais em termos de matéria orgânica, o que pode determinar alterações na quantidade e distribuição de poros. Carvalho et al. (1999) observaram que em um Podzólico VermelhoAmarelo, sob diferentes sistemas de preparo do solo, a maior retenção de água ocorreu no plantio direto devido à manutenção da matéria orgânica.

Não houve diferença entre os sistemas de manejo estudados em relação ao índice $\mathrm{S}$ na camada de 0 a 0,05 m. Como pode ser constatado (Quadro 4), nenhum tratamento teve valor de $\mathrm{S}$ menor que 0,035 , podendose, dessa forma, afirmar que esse solo possui boa qualidade física para o crescimento de plantas. Entretanto, resultados diferentes foram obtidos por Silva et al. (2008), que apresentaram valores de S menores que 0,035, possuindo assim uma estrutura física degradada, concordando com Dexter (2004).

$\mathrm{O}$ SC teve valor de índice $\mathrm{S}$ maior na camada de 0 a $0,05 \mathrm{~m}$, o que se deve ao revolvimento do solo e, consequentemente, ao aumento de poros de maior diâmetro; o mesmo não ocorreu na camada inferior. Segundo Dexter (2004), a redução do S nas áreas cultivadas pode estar associada à diminuição do pico da distribuição de frequência de poros, resultando em um "achatamento" vertical da curva de retenção de água, pela redução dos poros estruturais.

O SDL teve o menor índice $\mathrm{S}$ em profundidade, mas demonstrou que se encontra em boas condições físicas para o crescimento radicular, pois não teve valor de S menor que 0,035.

No quadro 5 estão apresentados os coeficientes de correlação de Pearson entre os indicadores da qualidade física do solo.

Foram observadas correlações significativas entre MO e Ds e entre MO e Pt. Resultados diferentes foram encontrados por Silva et al. (2008). A matéria orgânica
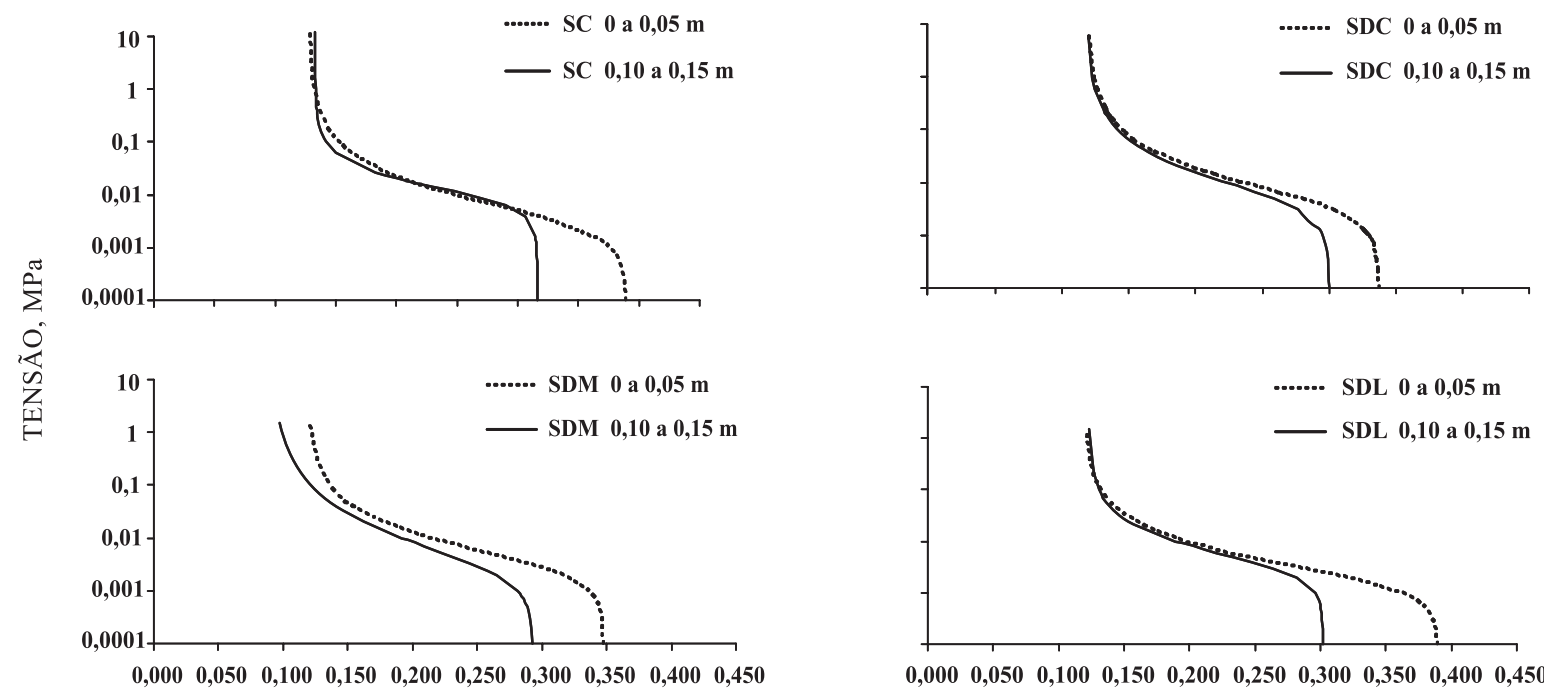

CONTEÚdO DE ÁGUA, $\mathrm{m}^{3} \mathrm{~m}^{-3}$

Figura 1. Curvas de retenção de água de um Latossolo Vermelho em sistemas de manejo. 
Quadro 4. Valores do índice S para os sistemas de manejo estudados nas camadas de 0,05 a $0,10 \mathrm{e}$ 0,15 a $0,20 \mathrm{~m}$ e a produtividade do milho

\begin{tabular}{|c|c|c|c|}
\hline Tratamento & Camada & Índice $S$ & Produtividade \\
\hline & $\mathrm{m}$ & & t ha-1 \\
\hline $\mathrm{SC}$ & $\begin{array}{l}0 \text { a } 0,05 \\
0,10 \text { a } 0,15\end{array}$ & $\begin{array}{l}0,056 \mathrm{~A} \mathrm{a} \\
0,039 \mathrm{AB} b\end{array}$ & $5280 \mathrm{~b}$ \\
\hline $\mathrm{SDC}$ & $\begin{array}{l}0 \text { a } 0,05 \\
0,10 \text { a } 0,15\end{array}$ & $\begin{array}{l}0,048 \mathrm{AB} a \\
0,047 \mathrm{AB} \text { a }\end{array}$ & 5920 a \\
\hline SDM & $\begin{array}{l}0 \text { a } 0,05 \\
0,10 \text { a } 0,15\end{array}$ & $\begin{array}{l}0,047 \mathrm{AB} \text { a } \\
0,045 \mathrm{AB} \text { a }\end{array}$ & $5489 a b$ \\
\hline SDL & $\begin{array}{l}0 \text { a } 0,05 \\
0,10 \text { a } 0,15\end{array}$ & $\begin{array}{l}0,041 \mathrm{AB} \text { a } \\
0,035 \mathrm{~B} \mathrm{~b}\end{array}$ & $5596 \mathrm{ab}$ \\
\hline
\end{tabular}

Médias seguidas da mesma letra não diferem estatisticamente pelo teste de Tukey a $5 \%$. SC: semeadura convencional; SDC: semeadura direta crotalária; SDM: semeadura direta milheto; SDL: semeadura direta lablabe.

é um dos fatores responsáveis pela formação e estabilidade de agregados; a formação de agregados na superfície provoca diminuição da densidade do solo. Aumentos na densidade do solo geralmente implicam diminuição na qualidade do solo para crescimento radicular e redução da aeração.

No que se refere à correlação entre $\mathrm{Ds}$ e Ma, é fato que, à medida que a densidade do solo aumenta, diminui a macroporosidade. Beutler et al. (2001) verificaram que o aumento da densidade do solo refletiu na redução da macroporosidade. Apesar de o valor da correlação entre Ds e Mi não ter sido significativo, apresentou magnitude relevante $(0,50)$ (Quadro 5). atributos físicos do solo. Assim, a presença de poros estruturais implica alto valor de $\mathrm{S}$, que é essencial para uma boa qualidade do solo.

Referente à correlação entre Ma e Mi significativa, é sabido que, à medida que uma dessas variáveis diminui, ocorre incremento da outra. O solo submetido ao cultivo intenso tende a perder a estrutura original pelo fracionamento dos agregados maiores em unidades menores, com consequente redução de macroporos e aumento de microporos (Carpenedo \& Mielniczuk, 1990).

Não foram observados valores significativos da correlação entre indicadores da qualidade do solo e produtividade. Como esses indicadores possuem valores considerados adequados, a baixa produtividade está, provavelmente, relacionada a outros fatores.

\section{CONCLUSÕES}

1. Na camada de 0 a $0,05 \mathrm{~m}$, os sistemas conservacionistas tiveram maior teor de matéria orgânica em relação ao SC.

2. Os maiores diâmetro médio ponderado e diâmetro médio geométrico dos agregados foram observados nos sistemas conservacionistas.

3. Nenhum dos sistemas de manejo estudados apresentou valores de índice $\mathrm{S}$ menores que 0,035.

4. Não foram observadas correlações significativas da correlação entre indicadores da qualidade do solo e produtividade.

Quadro 5. Coeficientes de correlação de Pearson entre os indicadores da qualidade física do solo MO (matéria orgânica), Ds (densidade do solo), Pt (porosidade total), Ma (macroporosidade), Mi (microporosidade), S (indice S) e Prod (produtividade do milho)

\begin{tabular}{|c|c|c|c|c|c|c|c|}
\hline Indicadores & MO & Ds & $\mathbf{P t}$ & Ma & Mi & $\mathbf{S}$ & Prod \\
\hline MO & - & $-0,6173^{*}$ & $0,9226^{* *}$ & 0,5019 & 0,2251 & 0,2357 & 0,2271 \\
\hline Ds & - & - & $-0,3777$ & $-0,7717 *$ & 0,5000 & $-0,6858^{*}$ & 0,0794 \\
\hline $\mathrm{PT}$ & - & - & - & 0,4980 & 0,2687 & $-0,0574$ & 0,2065 \\
\hline $\mathrm{Ma}$ & - & - & - & - & $-0,6667 *$ & 0,3523 & $-0,0394$ \\
\hline $\mathrm{Mi}$ & - & - & - & - & - & $-0,2524$ & 0,2626 \\
\hline $\mathrm{S}$ & - & - & - & - & - & - & $-0,0200$ \\
\hline Prod & - & - & - & - & - & - & - \\
\hline
\end{tabular}

*** Significativo a 1 e $5 \%$, respectivamente, pelo teste $\mathrm{t}$.

A correlação entre Ds e S foi significativa. De acordo com Dexter (2004), a declividade S da curva de retenção da água do solo no ponto de inflexão deve-se, principalmente, à porosidade microestrutural; portanto, o índice $\mathrm{S}$ avalia muitos dos principais

\section{LITERATURA CITADA}

ALBUQUERQUE, J.A.; ARGENTON, J. \& BAYER, C. Relação de atributos do solo com a agregação de um Latossolo Vermelho sob sistemas de preparo e plantas de verão para cobertura do solo. R. Bras. Ci. Solo, 29:415-424, 2005. 
ANDRIOLI, I. \& CENTURION, J.F. Levantamento detalhado dos solos da Faculdade de Ciências Agrárias e Veterinárias de Jaboticabal. In: CONGRESSO BRASILEIRO DE CIÊNCIA DO SOLO, Brasília, 1999. Anais... Brasília, Sociedade Brasileira de Ciência do Solo, 1999. p.1-4.

ARAÚJO, M.A.; TORMENA, C.A. \& SILVA, A.P. Propriedades físicas de um Latossolo Vermelho distrófico cultivado sob mata nativa. R. Bras. Ci. Solo, 28:337-345, 2004.

BEUTLER, A.N.; SILVA, N.L.N.; CURI, N.; FERREIRA, M.M.; CRUZ, J.N. \& PEREIRA FILHO, I.A. Resistência a penetração e permeabilidade de Latossolo Vermelho distrófico típico sob sistemas de manejo na região dos cerrados. R. Bras. Ci. Solo, 25:167-177, 2001.

BLAKE, G.R. \& HARTGE, K.H. Bulk density. In: KLUTE, A., ed. Methods of soil analysis. 2.ed. Madison, American Society of Agronomy, 1986. p.363-375.

BOER, C.A.; ASSIS, R.L.; SILVA, G.P.; BRAZ, A.J.B.P.; BARROSO, A.L.L.; CARGNELUTTI FILHO, A. \& PIRES, F.R. Ciclagem de nutrientes por plantas de cobertura na entressafra em um solo de Cerrado. Pesq. Agropec. Bras., 42:1269-1276, 2007.

CARPENEDO, V. \& MIELNICZUK, J. Estado de agregação e qualidade de agregados de Latossolos Roxos, submetidos a diferentes sistemas de manejo. R. Bras. Ci. Solo, 14:99105,1990

CARVALHO, E.J.M.; FIGUEIREDO, M.S. \& COSTA, L.M. Comportamento físico-hídrico de um Podzólico VermelhoAmarelo câmbico fase terraço sob diferentes sistemas de manejo. Pesq. Agropec. Bras., 34:257-265, 1999.

CENTURION, J.F. \& DEMATÊ, J.L.I. Efeitos de sistemas de preparo nas propriedades físicas de um solo sob cerrado cultivado com soja. R. Bras. Ci. Solo, 9:263-266, 1985.

COSTA, F.S.; ALBUQUERQUE, J.A.; BAYER, C.; FONTOURA, S.M.V. \& WOBETO, C. Propriedades físicas de um Latossolo Bruno afetados pelos sistemas plantio direto e preparo convencional. R. Bras. Ci. Solo, 27:527$535,2003$.

CRUZ, A.C.R.; PAULETTO, E.A. \& FLORES, C.A. Atributos físicos e carbono orgânico de um Argissolo Vermelho sob sistemas de manejo. R. Bras. Ci. solo, 27:1105-1112, 2003.

DANIELSON, R.E. \& SUTHERLAND, P.L. Porosity. In: KLUTE, A., ed. Methods of soil analysis: Physical and mineralogical methods. 2.ed. Madison, American Society of Agronomy, 1986. Part 1. p.443-461.

DEXTER A.R. Soil physical quality: Part II. Friability, tillage, tilth and hard-setting. Geoderma, 120:215-225, 2004

ESPINDOLA, J.A.A.; GUERRA, J.G.M.; ALMEIDA, D.L.; TEIXEIRA, M.G. \& URQUIAGA, S. Decomposição e liberação de nutrientes acumulados em leguminosas herbáceas perenes consorciadas com bananeiras. R. Bras. Ci. Solo, 30:321-328, 2006.

FURLANI, C.E.A.; LOPES, A. \& SILVA, R.P. Avaliação de semeadora-adubadora de precisão trabalhando em três sistemas de preparo do solo. Eng. Agríc., 25:458-464, 2005.
GARDNER, W.H. Water content. In: KLUTE, A., ed. Methods of soil analysis: Physical and mineralogical methods. 2.ed. Madison, American Society of Agronomy, 1986. Part 1. p.493-544.

GEE, G.W. \& BAUDER, J.W. Particle-size Analysis. In: KLUTE, A., ed. Methods of soil analysis: Physical and mineralogical methods. 2.ed. Madison, American Society of Agronomy, 1986. Part 1. p.383-411.

GIACOMINI, S.J.; AITA, C.; VEDRUSCOLO, E.R.O.; CUBILLA, M.; NICOLOSO, R.S. \& FRIES, M.R. Matéria seca, relação $\mathrm{C} / \mathrm{N}$ e acúmulo de nitrogênio, fósforo e potássio em misturas de plantas de cobertura de solo. R. Bras. Ci. Solo, 27:325-334, 2003.

KEMPER, W.D. \& CHEPIL, W.S. Size distribution of aggregates In: BLAKE, C.A.; EVANS, D.D.; WHITE, J.L.; ENSMINGER, L.E. \& CLARK, F.E., eds. Methods of soil analysis: Physical and mineralogical properties, including statistics of measurement and sampling. Madison, American Society of Agromony, 1965. p.499-510.

KEMPER, W.D. \& ROSENAU, R.C. Aggregate stability and size distribution. In: KLUTE, A., ed. Methods of soil analysis: Physical and mineralogical methods. 2.ed. Madison, American Society of Agromony, 1986. Part.1. p.499-509.

KLEIN, V.A. Propriedades físico-hídrico-mecânicas de um Latossolo Roxo, sob diferentes sistemas de uso e manejo. Piracicaba, Escola Superior de Agricultura Luiz de Queiroz, 1998. 150p. (Tese de Doutorado)

KLUTE, A. Water retention: Laboratory methods. In: KLUTE, A.. ed. Methods of soil analysis: Physical and mineralogical methods. 2.ed. Madison, America Society of Agronomy, 1986. p.635-660.

LETEY, J. Relationship between soil physical properties and crop production. Adv. Soil Sci., 1:277-294, 1985.

MARCHÃO, R.L.; SANTOS JUNIOR, J.D.G.; SILVA, E.M.; SÁ, M.A.C.; BALBINO, L.C.; VILELA, L. \& BECQUER, T. Parâmetro 'S' e intervalo hídrico ótimo em Latossolo Vermelho sob sistemas de integração lavoura-pecuária no Cerrado. In: CONGRESSO BRASILEIRO DE CIÊNCIA DO SOLO, 31., Gramado, 2007. Anais... Gramado, Sociedade Brasileira de Ciência do solo, 2007.

OLIVEIRA, F.C.; MATIAZZO, M.E.; MARCIANO, C.R. \& ROSSETO, R. Efeitos de aplicações sucessivas de lodo de esgoto em Latossolo Amarelo distrófico cultivado com cana-de-açúcar: Carbono orgânico, condutividade elétrica, pH e CTC. R. Bras. Ci. Solo, 26:505-519, 2002.

RAIJ, B.van; QUAGGIO, J.A.; CANTARELLA, H.; FERREIRA, M.; LOPES, A.S. \& BATAGLIA, O.C. Análise química do solo para fins de fertilidade. Campinas, Fundação Cargill, 1987. 170p.

SCHAFER, M.J.; REICHERT, J.M.; REINERT, D.J. \& CASSOL, E.A. Erosão em entressulcos em diferentes preparos e estados de consolidação do solo. R. Bras. Ci. Solo, 25:431441, 2001. 
SILVA, F.F.; FREDDI, O.S.; CENTURION, J.F.; ARATANI, R.G.; ANDRIOLI, F.F. \& ANDRIOLI, I. Propriedades físicas de um Latossolo Vermelho cultivado no sistema plantio direto. Irriga, 13:191-204, 2008.

SOUZA NETO, E.L.; ANDRIOLI, I.; BEUTLER, A.N. \& CENTURION, J.F. Atributos físicos do solo e produtividade de milho em resposta a culturas de pré-safra. Pesq. Agropec. Bras., 43:255-260, 2008.

TORMENA, C.A.; SILVA, A.P.; IMHOFF, S.C. \& DEXTER, A.R. Quantification of the soil physical quality of a tropical Oxisol using the S index. Sci. Agric., 65:56-60, 2008. van GENUCHTEN, M.T.A. A closed - form equation for predicting the hydraulic conductivity of insaturated soils. Soil Sci. Soc. Am. J., v.44, p.892-897, 1980.

WENDLING, B.; JUCKSCH, I.; MENDONÇA, E.S. \& NEVES, J.C.L. Carbono orgânico e estabilidade de agregados de um Latossolo Vermelho sob diferentes manejos. Pesq. Agropec. Bras., 40:487-494, 2005.

WIETHOLTER, S.; BEM, J.R.; KOCHHANN, R.A. \& POTTKER, D. Fósforo e potássio no solo no sistema plantio direto. In: NUERNBERG, N.J., ed. Conceitos e fundamentos do sistema plantio direto. Lages, Sociedade Brasileira de Ciência do Solo, 1998. p.121-149. 